\title{
An Image Processing Architecture to Exploit I/O Bandwidth on Reconfigurable Computers
}

\author{
Miaoqing Huang ${ }^{1}$, Olivier Serres ${ }^{1}$, Sergio Lopez-Buedo ${ }^{1 \S}$, Tarek El-Ghazawi ${ }^{1}$ and Greg Newby ${ }^{2}$ \\ ${ }^{1}$ Department of Electrical and Computer Engineering, The George Washington University \\ \{mqhuang,serres,sergiolb,tarek\}@gwu.edu \\ ${ }^{2}$ Arctic Region Supercomputing Center, University of Alaska Fairbanks \\ newby@arsc.edu
}

\begin{abstract}
FPGA devices in Reconfigurable Computers (RCs) allow datapath, memory, and processing elements (PEs) to be customized in order to achieve very efficient algorithm implementations. However, the maximum speedup on RCs is bounded by the bandwidth available between $\mu P \mathrm{~s}$ and FPGA hardware accelerators. In this paper, an image processing architecture is presented to fully exploit this bandwidth for achieving the maximum possible speedup. This architecture can be used to implement any convolution operation between an image and a kernel, and comprises four fully pipelined components: a line buffer, a data window, an array of PEs and a data concatenating block. Multiple image processing algorithms have been successfully implemented using this architecture, such as digital filters, edge detectors, and image transforms. In all cases, the maximum throughput is upper-bounded by the $\mu P$-FPGA I/O bandwidth, regardless of the complexity of the algorithm. This end-to-end throughput has been measured to be $1.2 \mathrm{~GB} / \mathrm{s}$ on Cray XD1 and $2.1 \mathrm{~GB} / \mathrm{s}$ on SGI RC100.
\end{abstract}

\section{INTRODUCTION}

Reconfigurable computers have been a hot area of research for several years. In particular, we define High-Performance Reconfigurable Computers (HPRCs) as parallel computers that resemble conventional High-Performance Computing (HPC) architectures, with added FPGA devices working as co-processors to the $\mu P(\mathrm{~s})$. Three systems, SRC-6, SGI Altix/RASC, and Cray XD1, are representatives of this definition. In these systems, the FPGA device is typically connected to the $\mu P$ through a proprietary and dedicated interconnect in order to avoid bus bottlenecks.

The clock rate of the FPGA device is generally one order of magnitude less than that of the $\mu P$. However, FPGA implementations can benefit from hardware parallelism in two

This work was supported in part by Arctic Region Supercomputing Center (ARSC) at University of Alaska Fairbanks.

$\S$ Visiting from the Computer Engineering Department of Universidad Autonoma de Madrid, Spain ways in order to beat $\mu P$ s. If the algorithm logic is able to retrieve multiple operands in one clock cycle and the operations on these operands are independent one of each other, we can instantiate multiple processing elements to process these operands. After the parallelism of hardware implementation is figured out based on the hardware environment and the algorithm itself, we can apply the other technique, pipelining, for designing the PE. Several stages are chained together and multiple operands can be processed concurrently, however residing at different stages. These two basic techniques are not exclusive to each other. The implementation of each stage in a pipeline may apply both pipelining and parallelism to achieve the best performance. This approach can provide performance improvements of 10 to 100 times. For some applications such as gene sequencing and cryptography, the performance is more than three orders of magnitude higher than using $\mu P \mathrm{~s}[1]$.

Low level image processing operators, such as digital filters, edge detectors and digital transforms are good candidates for hardware implementation. However, most of the previous works, e.g. [2, 3, 4], only focused on the algorithms itself and did not consider the platform characteristics as a factor in the design. In this paper, we present a novel hardware image processing architecture that is parameterized by the data transfer channel between $\mu P$ and FPGA subsystems on RC platforms. The architecture comprises fully pipelined components and is able to exploit the I/O bandwidth on those systems.

\section{BASIC IMAGE PROCESSING ALGORITHMS}

Most complex image processing applications, such as classification, feature extraction, pattern recognition and multiscale signal analysis, consist of multiple stages. Generally speaking, the very first step is applying basic techniques, such as digital filters and edge detectors, for facilitating the processing of the remaining steps [5]. In this paper, we present an architecture that can be efficiently adopted to implement those basic image processing algorithms.

Most of those basic algorithms involve the convolution 


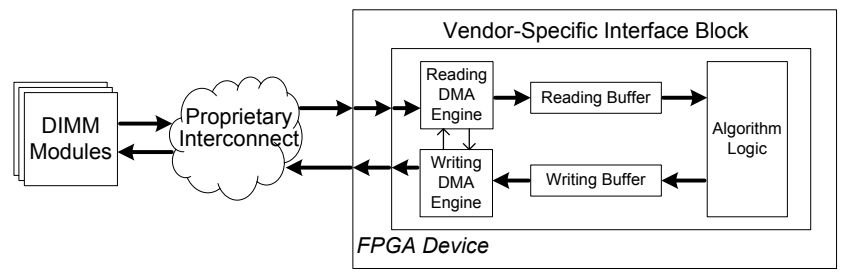

Fig. 1. Streaming Data Transfer Mode on Cray XD1.

process between the image and a kernel. Convolution provides a way of 'multiplying together' two arrays of numbers, generally of different sizes, but of the same dimensionality, to produce a third array of numbers of the same dimensionality. This can be used in image processing to implement operators whose output pixel values are simple linear combinations of certain input pixel values. If the image has $M$ rows and $N$ columns, and the kernel has $m$ rows and $n$ columns, then the size of the output image will have $M-m+1$ rows and $N-n+1$ columns. Mathematically we can write the convolution between the image $I$ and kernel $K$ as:

$$
O(i, j)=\sum_{k=1}^{m} \sum_{l=1}^{n} I(i+k-1, j+l-1) K(k, l) .
$$

Most digital filters or edge detectors are very straightforward and perform one stage of convolution. However, some operators take multiple kernels and stages before producing the result. The Compass Edge Detector, which not only estimates the local edge gradient magnitude but also estimates the edge orientation of the input image, applies a set of (in general 8) convolution masks, each of which is sensitive to one edge at a different orientation, and takes the response of the mask that yields the maximum local edge gradient magnitude. Particularly, the Canny Edge Detector comprises four stages, Gaussian Smoothing, Compass Edge Detecting, Non-maximum Suppression, and Edge Tracing \& Thresholding, in which each stage takes the output from the preceding stage and feeds its output to the following stage. By chaining these four stages into a pipeline, the hardware implementation is able to yield one pixel of the output image every clock cycle under ideal circumstances.

In general, those basic image processing algorithms allow the original image to be split into blocks of size $m \times n$ and these blocks can be processed independently. This feature lends them good candidates of hardware implementation to explore their intrinsic parallelism for reaching the upper-bound of I/O bandwidth.

\section{THE STREAMING DATA TRANSFER MODE}

The end-to-end throughput of hardware-accelerated applications is upper-bounded by the data transfer rate of the I/O between the $\mu P$ and FPGA subsystems on RC platforms. If
Table 1. I/O parameters on Cray XD1 and SGI RC100.

\begin{tabular}{c|c|c|c}
\hline \multirow{2}{*}{} & \multirow{2}{*}{ Bus Width } & \multicolumn{2}{|c}{ Max I/O bandwidth } \\
\cline { 3 - 4 } & & Theoretical & Practical \\
\hline Cray XD1 & $64-$ bit & $1.6 \mathrm{~GB} / \mathrm{s}$ & $1.2 \mathrm{~GB} / \mathrm{s}$ \\
\hline SGI RC100 & 128 -bit & $3.2 \mathrm{~GB} / \mathrm{s}$ & $2.1 \mathrm{~GB} / \mathrm{s}$ \\
\hline
\end{tabular}

we chain the I/O and hardware design into a pipeline, the ideal scenario happens when data run through the pipeline as a stream. We call this data transfer mode Streaming Data Transfer Mode, shown in Figure 1 for Cray XD1. Two DMA engines work in parallel to retrieve raw data blocks from and return result data blocks to the main memory. Under ideal circumstances, the reading DMA engine receives one raw data block from the input channel and the writing DMA engine puts one result data block to the output channel every clock cycle. Both Cray XD1 and SGI RASC RC100 provide the facilities and services for implementing the Streaming Data Transfer Mode in FPGA devices. Table 1 lists the I/O characteristics on both platforms.

\section{IMAGE PROCESSING ARCHITECTURE}

In Figure 1 we put the algorithm logic into a pipeline comprising I/O, DMA engines and reading/writing buffers. As one stage of the overall pipeline, the design of algorithm logic is influenced by the characteristics of other components in the same pipeline. In particular, the bus width and the bandwidth of I/O become two key parameters of the algorithm logic design.

In all the implementations, we assume that the image is in 8-bit gray level format. Furthermore, pixels in the original image are delivered into the algorithm logic in a stream, starting with the pixel of the top-left corner, ending with the pixel of the bottom-right corner. Because the bus width of the interconnect fabric is multiple-byte wide in general, several pixels are fed into the algorithm logic in same clock cycle. To maximize the throughput of the overall architecture, the algorithm logic has to be able to perform the operations of multiple pixels concurrently and take new data input every clock cycle. In the following discussion, we assume that the image size is $M \times N$, the kernel size is $m \times n$ and the bus width of the interconnect fabric is $D$ bytes.

The diagram of the algorithm logic is shown in Figure 2. The architecture consists of four components, one Line Buffer, one Data Window, an array of PEs and the Data Concatenating Block.

The quantity of PEs is $x$, which depends on the bus width of the interconnect network and the particular algorithm. For all the digital filters and edge detectors, the number of PEs is $D$. There are $\frac{D}{2}$ PEs for DWT, however. Every $\mathrm{PE}$ is fully pipelined and is able to take new input, one block 

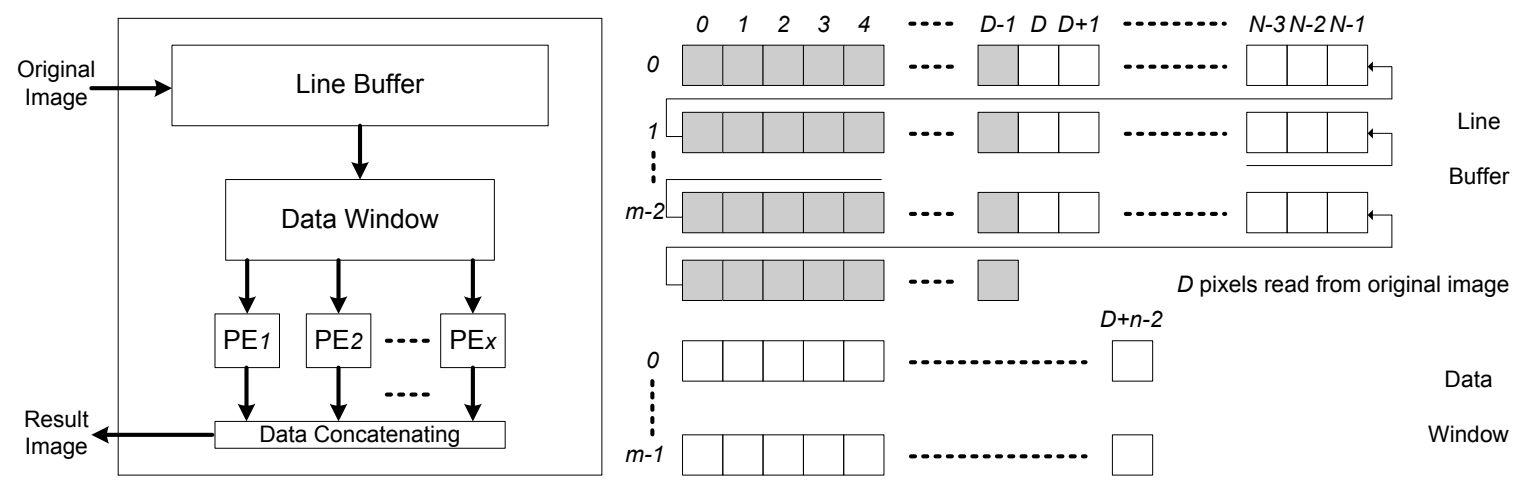

Fig. 2. The Algorithm Logic Diagram.

of $m \times n$ pixels, every clock cycle. The function of PEs depends on the implemented algorithm, and the output of one $\mathrm{PE}$ is the intensity of one pixel in the result image.

Data Window is a two-dimension register array of $m \times$ $(n+D-1)$ in charge of providing image blocks to the downstream PEs. Analogously, the Data Window scans the original image from left to right and from top to bottom with an horizontal stride of $D$ pixels and a vertical stride of 1 pixel. Once the data window receives a valid input, one block of $m \times D$ pixels, it does a $D$-byte left shift for all rows with the input tailing the right most $n-1$ columns.

Line Buffer is a two-dimension register array of $(m-$ 1) $\times N$. The original image is transferred into the FPGA as a stream. However, PEs request image blocks that spread on different rows. The purpose of the line buffer is to keep all pixels in registers until one $m \times D$ block forms. One $m \times D$ block, shown in gray color in Figure 2, comprises the new arrived $D$ pixels and another $(m-1) \times D$ pixels that reside at the head of every row of the line buffer. Every time the line buffer receives $D$ pixels of the original image, it performs two actions simultaneously. The first action is to deliver the new formed image block to the data window. The second action is to do a $D$-byte left shift in a zigzag form, in which two neighbor rows are linked together by connecting their tail and head.

For most of these cases, the design of the Data Concatenating Block is straightforward because it generally concatenates the outputs of upstream PEs together. However, this block needs to perform a more complicated function for some cases. For example, in a 9-point DWT, every other pixel will generate one high coefficient and one low coefficient. If these high and low coefficients are transferred back to main memory in an interleaved way, the $\mu P$ has to spend extra time to reorganize them and thus decrease the overall performance. In this case, the data concatenating block can wait for the end of DWT processing of one line and then reorder the coefficients before sending them back to host.

In general, the four components in Figure 2 form a pipeline chain and each of them is fully pipelined as well.
This architecture is able to accept $D$ pixels of the original image every clock cycle and output $D$ pixels of the result image every clock cycle at the same time. In case of a multiplestage algorithm, such as the four-stage Canny Edge Detector, various stages can be chained together and each stage consists of these components with different parameters and functionalities.

Line Buffer, Data Window and Data Concatenating Blocks should be implemented in registers instead of BRAM because we need to access all the elements concurrently. The design of PEs is beyond the scope of this paper. However, two techniques are frequently applied to facilitate the hardware design. (1) Use fixed-point instead of floatingpoint operation. (2) In the design of the Compass Edge Detector, rotate pixels in the same block instead of designing eight different operators to cover various orientations.

\section{IMPLEMENTATION AND RESULTS}

Following is a list of the basic image algorithms that have been implemented using the purposed architecture.

$\diamond$ Digital Filter: Mean Filter, Median Filter, Gaussian Smoothing Filter, Conservative Smoothing Filter, and Unsharp Filter.

$\diamond$ EDGE Detector: Sobel Edge Detector, Prewitt Gradient Edge Detector, Compass Edge Detector (Magnitude and Orientation), and Canny Edge Detector. Compass Edge Detector comprises Sobel Edge, Prewitt, Robinson, and Krisch.

$\diamond$ IMAGE TRANSFORM: Discrete Wavelet Transform.

For most of the algorithms in the list, the kernel size is $3 \times 3$. However, the kernel size in the Gaussian Smoothing Filter is $5 \times 5$, which is the same size of the filter used in the first stage of the Canny Edge Detector. DWT is the CDF 9/7 wavelet transform, which is the one used in the JPEG-2000 standard. In the design of the PE for the Compass Edge Detector, the original $3 \times 3$ block is rotated eight times, representing eight different orientations, $0^{\circ}, 45^{\circ}, \cdots$, $315^{\circ}$ respectively. These eight variants of the original pixel 
Table 2. Performance improvement of selected image processing algorithms on two reconfigurable computers ${ }^{\dagger}$.

\begin{tabular}{|c|c|c|c|c|c|c|c|c|}
\hline \multirow{3}{*}{ Algorithm Name } & \multicolumn{5}{|c|}{ End-to-end Throughput (MB/s) } & \multicolumn{3}{|c|}{ Resource Utilization } \\
\hline & \multirow{2}{*}{$\mu P^{\ddagger}$} & \multicolumn{2}{|c|}{ Cray XD1 } & \multicolumn{2}{|c|}{ SGI RASC } & \multicolumn{2}{|c|}{ Cray XD1 } & \multirow{2}{*}{$\begin{array}{c}\text { SGI RASC }^{\sharp} \\
\text { Slices }\end{array}$} \\
\hline & & XD1 & Speedup & RASC & Speedup & Slices & Multipliers & \\
\hline Median Filter & 22.80 & 1,196 & 52.5 & 2,124 & 93.2 & $6,752(28 \%)$ & $0(0 \%)$ & $16,341(18 \%)$ \\
\hline Gaussian Smoothing Filter & 12.80 & 1,196 & 93.4 & 2,124 & 165.9 & $11,403(48 \%)$ & $200(86 \%)$ & $58,143(65 \%)$ \\
\hline Sobel Edge Detector & 100.70 & 1,196 & 11.9 & 2,124 & 21.1 & $5,070(21 \%)$ & $0(0 \%)$ & $13,628(15 \%)$ \\
\hline P.C. Edge Detector ${ }^{\S}$ & 3.45 & 1,196 & 346.7 & 2,124 & 615.7 & $8,603(36 \%)$ & $0(0 \%)$ & $20,217(22 \%)$ \\
\hline Canny Edge Detector & 2.20 & 1,196 & 543.6 & 2,124 & 965.5 & $15,015(63 \%)$ & $200(86 \%)$ & $78,684(88 \%)$ \\
\hline DWT (CDF 9/7) & 89.10 & 1,196 & 13.4 & 2,124 & 23.8 & $14,798(62 \%)$ & $72(31 \%)$ & $44,373(49 \%)$ \\
\hline
\end{tabular}

${ }^{\dagger}$ The FPGA devices on XD1 and RASC are Xilinx XC2VP50FF1152-7 and XC4VLX200FF1513-10 respectively.

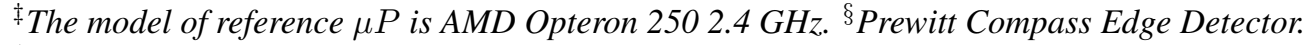

$\sharp$ On XC4VLX200 FPGA device, LUTs are used to build multipliers instead of built-in multipliers on XC2VP50.

block are processed by eight instances of the same operator concurrently. The outputs of these operators are compared to get the orientation which yields the maximal magnitude. Compass edge detection is only one of the four stages that compose the Canny Edge Detector. The hardware implementation of every stage can follow the architecture shown in Figure 2. By concatenating these four stages together, we obtain a fully pipelined hardware design of this edge detector, which is able to take 8 and 16 pixels each clock cycle on Cray XD1 and RASC, respectively.

All the bitstreams we generated run at $200 \mathrm{MHz}$. The end-to-end throughput, including all the data transfer and data processing, is the same for all algorithms, equal to the available I/O bandwidth. Table 2 lists the performance improvement of some algorithms' hardware implementation on both platforms compared to a software version running on the $\mu P$ of Cray XD1, Opteron $2502.4 \mathrm{GHz}$. The reported slice utilization comprises the algorithm logic, the supporting logic and the vendor-specific interface block. The supporting logic and the vendor-specific interface block occupy approximately 4,700 slices on Cray XD1 and 11,800 slices on SGI RC100. The algorithm logic itself on RASC generally occupies two times more slices than its counterpart on Cray XD1 platform because the I/O on RASC is twice wider than that of Cray XD1. Moreover, the built-in multipliers are used on Cray XD1. On RASC, LUTs are used instead because the XC4VLX200 FPGA device has a very limited number (96) of built-in multipliers. This factor contributes to the slice utilization increase on RASC for those algorithms that perform many multiplications, for instance, the Gaussian Smoothing Filter.

\section{CONCLUSION}

We have presented a hardware image processing architecture that is able to exploit the $\mu P$-FPGA bandwidth on re- configurable computers. By linking together I/O and processing logic in a pipeline, the maximum possible throughput is achieved regardless of the algorithm complexity. This architecture is designed for implementing those basic image processing algorithms which perform convolution or convolution-like operations between the original image and a kernel. The algorithms implemented include digital filters, edge detectors and the DWT. For all cases, the end-toend throughput has been found to be determined by the I/O bandwidth, $1.2 \mathrm{~GB} / \mathrm{s}$ on Cray XD1 and 2.1 GB/s on SGI RASC. The proposed image architecture can be applied to any other RC platform, the only point to be considered is that the final throughput will be fixed by the I/O bandwidth of the selected RC machine.

\section{REFERENCES}

[1] P. Saha, E. El-Araby, M. Huang, T. El-Ghazawi, and et al., "Portable library development for reconfigurable computing systems," in Proc. The 2007 Reconfigurable Systems Summer Institute (RSSI'07), July 2007.

[2] M. A. de Barros and M. Akil, "Low level image processing operators on FPGA: implementation examples and performance evaluation," in Proc. the 12th IAPR Intarnational Conference on Pattern Recognition, vol. 3, Oct. 1994, pp. 262-267.

[3] V. Muthukumar and D. V. Rao, "Image processing algorithms on reconfigurable architecture using HandelC," in Proc. IEEE EUROMICRO Systems on Digital System Design (DSD'04), vol. 1, Aug. 2004, pp. 218-226.

[4] K. T. Gribbon, D. G. Bailey, and C. T. Johnston, "Using design patterns to overcome image processing constraints on FPGAs," in Proc. Third IEEE International Workshop on Electronic Design, Test and Applications, 2006(DELTA 2006), Jan. 2006, pp. $47-56$.

[5] R. C. Gonzalez and R. E. Woods, Digital Image Processing (3rd Edition). Upper Saddle River, NJ: Prentice Hall, Aug. 2007. 